\title{
Comparison of the use of comprehensive point-of-care test panel to conventional laboratory process in emergency department
}

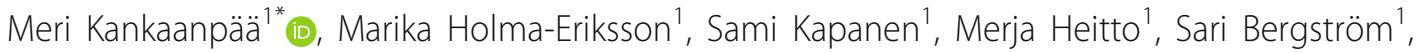 \\ Leila Muukkonen ${ }^{2}$ and Veli-Pekka Harjola ${ }^{1}$
}

\begin{abstract}
Background: In this study, we hypothesized that point of care testing (POCT) would reduce length of stay (LOS) in emergency department (ED) when compared to central laboratory testing and be a factor in patient discharge destination.

Methods: A single centre observational study was performed in ED non-ambulatory patients. Blood testing was performed either with POC instruments for blood gases and chemistry panel, full blood count, and CRP, or at central laboratory, or as a combination of both. Blood draw and POCTs were performed by experienced nurses.

Results: During the 4-week study period, 1759 patients underwent sample testing (POCT: $n=160$, central lab: $n=951$; both $n=648)$. Median waiting time for blood sampling was 19 min less in POCT than central laboratory (0:52 (95\% confidence interval (Cl) 0:46-1:02) vs. 1:11 (95\% Cl 1:05-1:14), $p<0.001)$. POCT results were available faster in both discharge groups, as expected. When imaging was not required, patients in POCT group were discharged home 55 min faster (4:57 (95\% Cl 3:59-6:17) vs. 5:52 (95\% Cl 5:21-6:35), $p=0.012$ ) and $1 \mathrm{~h} 22$ min faster when imaging was performed (5:48 (95\% Cl 5:26-6:18) vs. 7:10 (95\% Cl 6:47-8:26), $p=0.010)$. Similar reduction in sampling time and LOS was not seen among those admitted to hospital.

Conclusions: POCT shortened the laboratory process and made results available faster than the central lab. This allowed patients to be discharged home quicker. Thus, with proper training and education of the ED care team, POCT can be used as an effective tool for improving patient flow.
\end{abstract}

Keywords: Laboratory testing, Point-of-care testing, Emergency department, Length of stay, Discharge

\section{Background}

High quality and patient-centered care requires early diagnosis, which is achieved by eliminating unnecessary pre- and post-analytical delays. Blood testing and diagnostic imaging are essential routines of ED, and especially blood testing is associated with prolonged length of stay [1]. The laboratory turn-around time for results from central laboratories (CL) can be over $60 \mathrm{~min}$,

\footnotetext{
* Correspondence: meri.kankaanpaa@helsinki.fi

${ }^{1}$ Emergency Medicine, University of Helsinki and Department of Emergency Medicine and Services, Helsinki University Hospital, Haartmaninkatu 4, PL 340, 00029 Helsinki, Finland

Full list of author information is available at the end of the article
}

compared to 10 to $15 \mathrm{~min}$ for point-of-care bedside testing (POCT).

Many studies on POCT, focused on selected tests and limited patient populations, have suggested reduced length of stay (LOS) [2-4]. A recent study by Singer et al. focusing on critical care patient population reported reduced LOS using similar iSTAT POCT equipment as used in this study [5]. Some have also reported that POCT strategy alone has not necessarily improved LOS or that it has had effect on only a certain group of patients [6]. Most studies on POCT focus on diagnostic accuracy $[7,8]$ instead of process improvement. The full benefit of POCT is acquired when it is implemented

(c) The Author(s). 2018 Open Access This article is distributed under the terms of the Creative Commons Attribution 4.0 International License (http://creativecommons.org/licenses/by/4.0/), which permits unrestricted use, distribution, and reproduction in any medium, provided you give appropriate credit to the original author(s) and the source, provide a link to the Creative Commons license, and indicate if changes were made. The Creative Commons Public Domain Dedication waiver (http://creativecommons.org/publicdomain/zero/1.0/) applies to the data made available in this article, unless otherwise stated. 
together with process redesign $[9,10]$. When properly used, POCT can result in a number of benefits in the quality and efficiency of care [11].

Focusing on the process management point of view, we hypothesized that POCT would reduce LOS in emergency department (ED) when compared to central laboratory testing and be a factor in patient discharge destination; home or hospital.

\section{Methods}

\section{Study design}

The study was performed as a prospective single centre study in a Finnish metropolitan hospital with approximately 61,000 annual visits at the hospital's ED. The patient population consists of specialty care adult patients, and outside office hours also primary care patients. Central laboratory is located at the same site but operated separately, with ED being one of their clients. Laboratory personnel take samples which are then transported via a pneumatic tube system for analysis.

This study focuses on non-ambulatory patients who needed blood sampling (Fig. 1). Urine, fecal and cerebrospinal fluid testing were excluded from the analysis. Also, only laboratory results ready before discharge were taken into account. The study period was 1 month, lasting from Dec 12, 2016 to Jan 11, 2017.

\section{Study protocol}

The central laboratory personnel validated the POCT instruments, and their diagnostic accuracy was agreed to be at a clinically acceptable level. Nurses were trained to take blood samples and use the POCT equipment. Each nurse went through two training sessions ( $2 \mathrm{~h}$ each), and did a skills demonstration test before qualification. The nurses were advised to take blood samples from patients during initial assessment and management. Physicians were informed about the project. POC testing was instructed to be the primary analysis method for each non-ambulatory patient. Central lab was used either alone, when samples were taken by a biomedical laboratory scientist, or in addition to POCT, when both samples were taken by an ED nurse. Both central lab and ED nurses operated $24 \mathrm{~h}$ a day, 7 days a week.

Blood testing was performed either with POC instruments iSTAT (Abbott) for blood gases and chemistry panel, and PocH-100i (Roche) for blood count, and Afinion (Alere) for CRP; or at central laboratory; or combination of both. Three iSTAT testing devices were used for sodium, potassium, creatinine, urea, ionized calcium, blood gas analysis and glucose. Troponin I was excluded from the protocol due to low detection limit. ISTAT devices were given out to three patient areas of 9 to 10 beds having two nurses each. No extra personnel resources were added.

The POCT results were sent electronically to the central laboratory database and reported through the electronic medical record system. Data were collected from hospital, laboratory and imaging databases. The search was limited to patients who visited the ED during the length of the project and whose laboratory and radiology tests were ordered by the ED.

\section{Outcome measures}

Waiting time from admission to laboratory sampling, diagnostic imaging (like $\mathrm{x}$-ray and CT scans) and discharge (LOS) was calculated. Discharge time point was

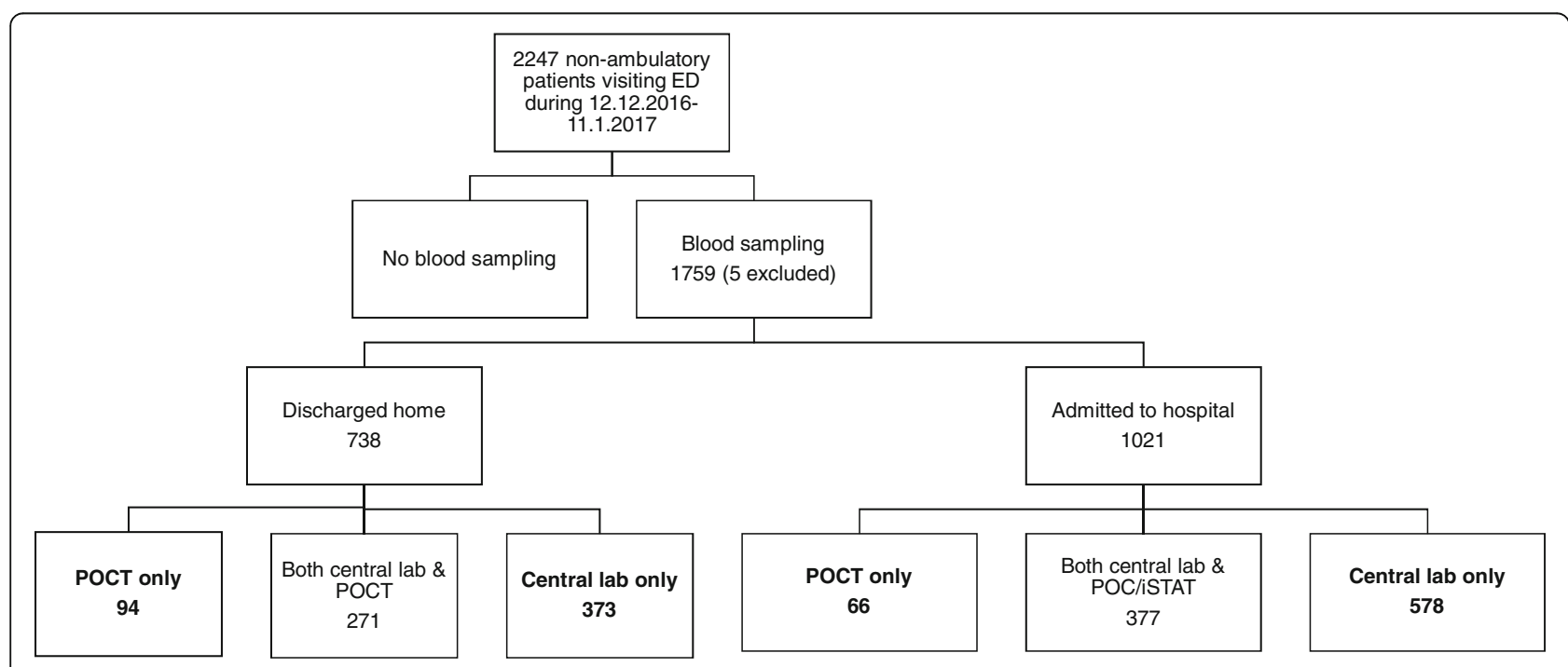

Fig. 1 Study patient population. The POCT only groups were compared to the Central lab only group 
when the patient left the ED, since it was not possible to identify the time the patient was ready for the discharge. One patient may have had multiple laboratory time stamps. The first sampling time per patient visit was identified, and laboratory results were considered ready when all results before discharge were ready. Results were often ready at different times due to multiple analytical laboratory devices used. The central laboratory process registered the actual sampling time, POCT sampling time was defined as the time when analysis started. Due to this, 5 to $10 \mathrm{~min}$ should be reduced from POCT admission to sampling time.

\section{Data analysis}

Median waiting times were calculated since data was positively skewed i.e. had a long right tail. Medians were presented with $95 \%$ confidence intervals $(95 \% \mathrm{CI})$ (Figs. 2 and 3), and 95\% CIs were also calculated. Patients discharged home were analyzed separately from patients admitted to hospital. Finally, patient groups including only POC tested patients were compared to those analyzed by central lab only. Patients having both central laboratory testing and POCT were excluded from the study in order to get better comparability between two testing options.

Mann \& Whitney U-test was used to test for differences in waiting time distributions between two subgroups, and results were presented as $p$-value. Statistical analyses were performed using SPSS computer software (SPSS Inc., Chicago, IL, USA). All interpretations are based on $\alpha=0.05$.

\section{Results}

Age and sex patterns were mainly similar in all subgroups. Patients discharged home were younger than those admitted to hospital ward and had more patients analyzed by POC only in relation to patients admitted to hospital (Table 1).

Compared to patients discharged home from ED, the ones admitted to hospital more often had infectious diseases and less often cardiopulmonary disease. The patients who required central laboratory testing had more often infectious diseases and less often abdominal complaints than the patients in POC group (Table 1) The group of patients having both POC and central laboratory testing (excluded from study) had a diagnosis distribution relatively similar to the group tested by central laboratory only. Approximately $70 \%$ of non-ambulatory patients went through radiological imaging during their stay in ED.

Figure 2 shows waiting times of patients discharged home. Median waiting time for blood sampling was $19 \mathrm{~min}$ less for POC patients when sampling was done by ED nurses, compared to central laboratory patients (0:52 (95\% CI 0:46-1:02) vs. 1:11 (95\% CI 1:05-1:14), $p<0.001)$. In actuality, the difference is even larger, since POC blood samples were taken 5 to $10 \mathrm{~min}$ before the first time stamp. Similar reduction in

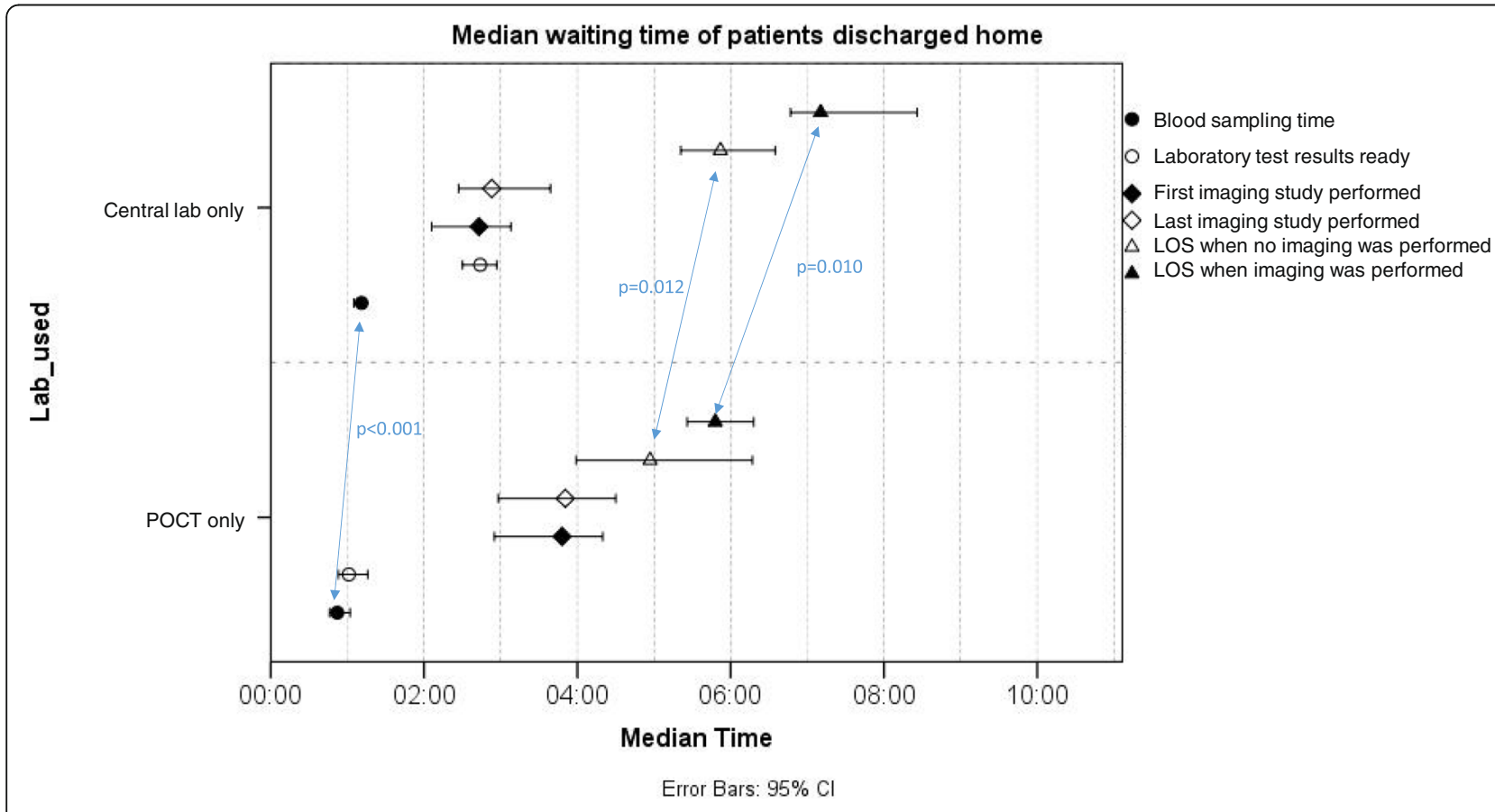

Fig. 2 Median waiting times from admission to blood sampling, laboratory results ready, imaging and discharge home with 95\% confidence intervals 


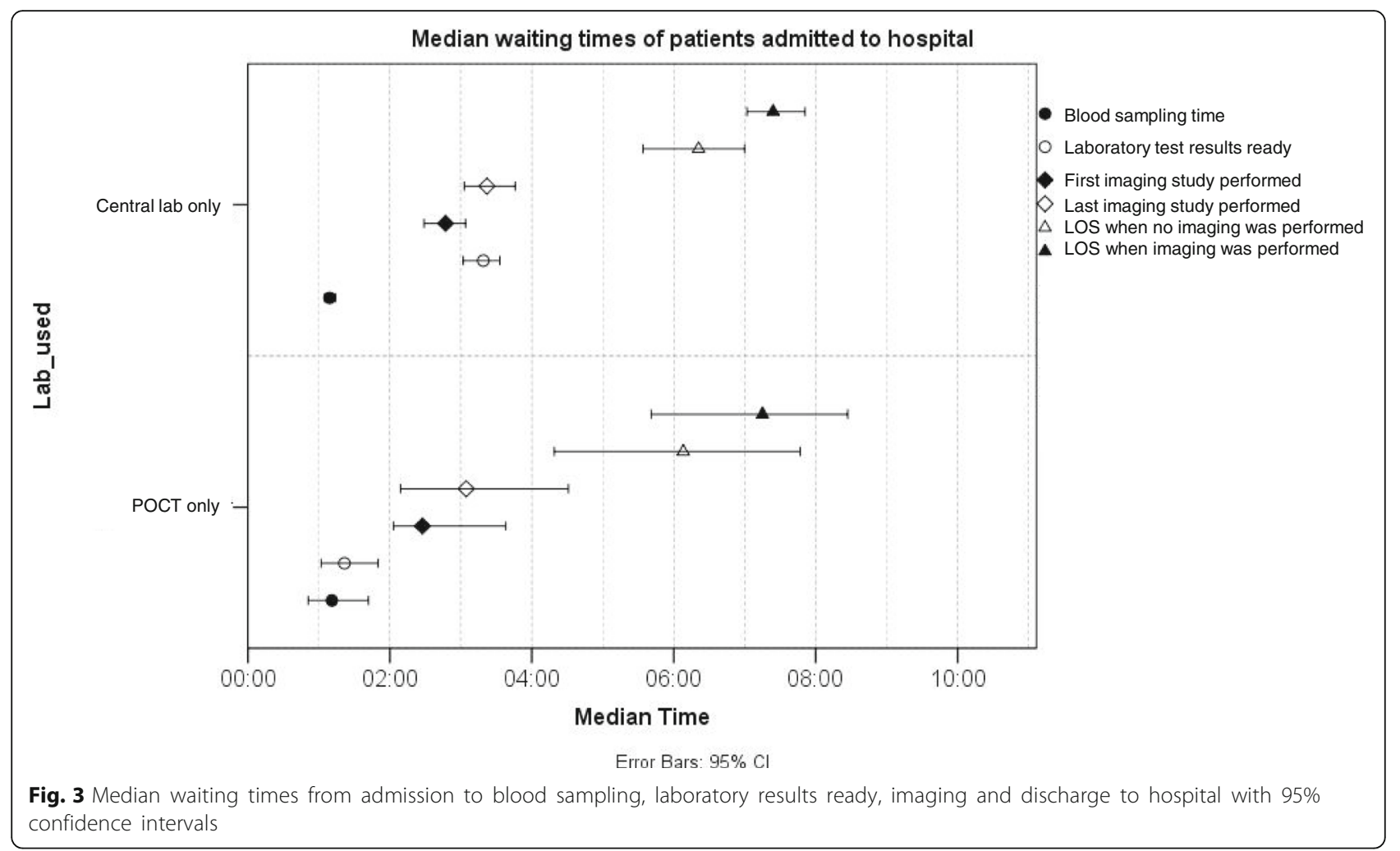

sampling waiting time was not seen in the patient group admitted to hospital (Fig. 3).

POCT results were available significantly faster in both discharge groups, as expected. POCT results were completed $1 \mathrm{~h} 1 \mathrm{~min}$ faster in the discharged home group (00:06 (95\% CI 0:05-0:07) vs. 1:07 (95\% CI 1:011:13), $p<0.001)$ and $1 \mathrm{~h} 39 \mathrm{~min}$ faster in the admitted to hospital group (0:06 (95\% CI 0:04-0:07) vs. 1:45 (95\% CI 1:33-1:57), $p<0.001)$. One hundred and-forty
POCT patients from total 160 had results ready in less than $15 \mathrm{~min}$. The 20 patients above $15 \mathrm{~min}$ had had multiple POC tests taken, which were completed at different times.

However, it was only the patients discharged home (Fig. 2), who were discharged $55 \mathrm{~min}$ faster without imaging (4:57 (95\% CI 3:59-6:17) vs. 5:52 (95\% CI 5:21-6:35), $p=0.012$ ) and $1 \mathrm{~h}$ and $22 \mathrm{~min}$ faster with imaging (5:48 (95\% CI 5:26-6:18) vs. 7:10 (95\% CI

Table 1 Baseline characteristics of study participants

\begin{tabular}{|c|c|c|c|c|}
\hline & \multicolumn{2}{|c|}{ Discharged home } & \multicolumn{2}{|c|}{ Admitted to hospital } \\
\hline & POCT only & Central lab only & POCT only & Central lab only \\
\hline Number of patients & 94 & 373 & 66 & 578 \\
\hline Mean age, years & 63 & 59 & 68 & 69 \\
\hline Females \% & $57 \%$ & $54 \%$ & $59 \%$ & $52 \%$ \\
\hline \multicolumn{5}{|l|}{ Diagnosis } \\
\hline Infection & $9(10 \%)$ & $88(24 \%)$ & $14(21 \%)$ & $233(40 \%)$ \\
\hline Cardiopulmonary & $24(26 \%)$ & $105(28 \%)$ & $12(18 \%)$ & $104(18 \%)$ \\
\hline Neurology & $16(17 \%)$ & $60(16 \%)$ & $10(15 \%)$ & $50(9 \%)$ \\
\hline Other & $10(11 \%)$ & $52(14 \%)$ & $7(11 \%)$ & $65(11 \%)$ \\
\hline Abdominal & 17 (18\%) & $28(8 \%)$ & $6(9 \%)$ & $53(9 \%)$ \\
\hline Musculoskeletal & $6(6 \%)$ & $21(6 \%)$ & $9(14 \%)$ & $32(6 \%)$ \\
\hline Unspecific complaint & $6(6 \%)$ & $9(2 \%)$ & $6(9 \%)$ & $26(4 \%)$ \\
\hline Alcohol & $6(6 \%)$ & $10(3 \%)$ & $2(3 \%)$ & $15(3 \%)$ \\
\hline
\end{tabular}


6:47-8:26), $p=0.010)$. Similar reduction in LOS was not seen among those admitted to hospital (Fig. 3).

\section{Discussion}

In our study, only those discharged home were fully able to benefit from POCT and were discharged $55 \mathrm{~min}$ (no imaging) or $1 \mathrm{~h} 22 \mathrm{~min}$ (imaging) earlier. The time from ED admission to sampling and results ready to discharge will not be reduced without changes in working practice. Patients' admittance to hospital/care unit may be delayed due to need for additional diagnosis, or lack of availability of hospital beds.

Analysis did not reveal any specific group with whom POCT cannot be used. The POCT group represented all diagnostic subgroups and the group having both POC and central lab testing was similar to those tested only by central lab.

The study results are in agreement with previous studies evaluating POCT impact on waiting times and LOS. Singer et al. evaluated POCT impact on critical care patients using iSTAT devices ( $\mathrm{Hb}$, hematocrit, TnI, lactate, BNP, INR). They reported a 33-min reduction in median LOS in patients admitted during office hours (not statistically significant) and an 87-min reduction in median LOS in patients presenting at all times who required a CT with IV contrast [5]. Jang et al. [12] compared ED length of stay when noncritical nonpediatric patients were assigned to a comprehensive point-of-care test to those with central laboratory testing. They reported a $22 \mathrm{~min}$ shorter median LOS compared to CL group (with a LOS approx. $6 \mathrm{~h}$ ) [12]. In our previous study with ambulatory patients, introduction of POCT reduced median LOS by $29 \mathrm{~min}$, and the Early Assessment Team (EAT) model reduced median LOS further by $17 \mathrm{~min}$. EAT consists of a consultant emergency physician and a nurse, aiming at define the need for laboratory testing and imaging fast in order to make process faster and safe for the patient. Altogether, the process was expedited by $46 \mathrm{~min}$ compared to original setup [9]. Lee-Lewandrowski et al. [13] evaluated LOS before and after implementation of POCT laboratory testing glucose, urine dipstick, hCG, and cardiac markers. They reported a 41-min decrease in patient LOS. Singer et al. [14] reported a 64-min reduction in LOS in a similar study [13].

Thus, with proper training and education of the ED care team, POCT can be used as an effective tool for managing patient flow in ED. However, best value for investment is received when process efficiency is also optimized. POCT together with efficient triage, senior consultant support and best care pathways would probably decrease LOS further.

\section{Limitations}

Patients were passively divided into each study group and not actively randomized. This may affect the comparability of groups, and it also resulted in relatively big differences in group sizes.

Influenza epidemic landed during project time, which challenged ED resources and may have had an impact on patient population characteristics. The number of inpatient beds is limited, and this affects the admitted to hospital group LOS.

\section{Conclusions}

The results of this study demonstrate that POCT provides laboratory results faster than the traditional central laboratory process. However, process improvement is needed in order to take advantage of this faster availability of laboratory results.

In this population, POCT shortened the length of stay in ED in patients discharged home. Further studies are needed to show whether achieving laboratory results earlier translates to improved patient safety.

\section{Abbreviations}

ED: Emergency department; LOS: Length of stay; POCT: Point-of-care testing

\section{Acknowledgements}

The central laboratory personnel and nursing staff of Jorvi Emergency Department, Helsinki University Hospital, are greatly appreciated for their invaluable contribution to sampling and performing POCT analyses. We thank Abbott Laboratories for providing unrestricted grant and POCT equipment for the study period.

\section{Funding}

The study was supported by unrestricted grant and point of care test equipment from Abbott Laboratories.

\section{Availability of data and materials}

The datasets used and/or analyzed during the current study are available from the corresponding author on reasonable request.

\section{Authors' contributions}

MK: collection and analysis of the data, evaluation of results, and writing of the manuscript. MH-E: planning and development of the POCT processes, and critical revision of the manuscript. SK: planning and development of the POCT processes, and critical revision of the manuscript. $\mathrm{MH}$ : planning and development of the POCT processes, and critical revision of the manuscript. SB: planning and development of the POCT processes, and critical revision of the manuscript. LM: validation of the POCT tests, development of POCT sampling, quality assessment, and critical revision of the manuscript. $\mathrm{V}-\mathrm{PH}$ : Preparation of the study protocol, evaluation of the results, and writing of the manuscript. All authors have read and approved the manuscript.

Ethics approval and consent to participate

This study was approved by institutional review board of the Helsinki University Hospital and the Department of Emergency Medicine and Services. Committee's reference number is $\$ 11$ HUS 1.3.2015.

Need for consent was waived by the IRB of the Department of Emergency Medicine and Services, Helsinki University Hospital.

Consent for publication

Not applicable.

Competing interests

The authors declare that they have no competing interests. 


\section{Publisher's Note}

Springer Nature remains neutral with regard to jurisdictional claims in published maps and institutional affiliations.

\section{Author details}

${ }^{1}$ Emergency Medicine, University of Helsinki and Department of Emergency Medicine and Services, Helsinki University Hospital, Haartmaninkatu 4, PL 340, 00029 Helsinki, Finland. ${ }^{2}$ Department of Clinical Chemistry and Haematology, Helsinki University Hospital, HUSLAB, Helsinki, Finland.

Received: 7 June 2018 Accepted: 8 November 2018

Published online: 19 November 2018

\section{References}

1. Kocher K, Meurer W, Desmond J, Nallamothu B. Effect of testing and treatment on emergency department length of stay using a National Database. Acad Emerg Med. 2012;19(5):525-34.

2. Asha S, Chan A, Walter E, Kelly P, Morton R, Ajami A, Wilson R, Honneyman D. Impact from point-of-care devices on emergency department patient processing times compared with central laboratory testing of blood samples: a randomised controlled trial and cost-effectiveness analysis. Emerg Med J. 2014;31(9):714-9.

3. Zenlander R, von Euler M, Antovic J, Berglund A. Point-of-care versus central laboratory testing of INR in acute stroke. Acta Neurol Scand. 2017;00:1-4.

4. Loten C, Attia J, Hullick C, Marley J, McElduff P. Point of care troponin decreases time in the emergency department for patients with possible acute coronary syndrome: a randomised controlled trial. Emerg Med J. 2010; 27:194-8.

5. Singer A, Williams J, Taylor M, Le Blanc D, Thode H. Comprehensive bedside point of care testing in critical ED patients: a before and after study. Am J Emerg Med. 2015;33(6):776-80.

6. Murray R, Leroux M, Sabga E, Palatnick W, Ludwig L. Effect of point of care testing on length of stay in an adult emergency department. J Emerg Med. 1999;17(5):811-4.

7. Mclntosh B, Vasek J, Taylor M, Le Blanc D, Thode H, Singer A. Accuracy of bedside point of care testing in critical emergency department patients. Am J Emer Med. 2017; in press.

8. Ivaska L, Niemelä J, Leino P, Mertsola J. Accuracy and feasibility of point-ofcare white blood cell count and C-reactive protein measurements at the pediatric emergency department. PLoS One. 2015;10(6):e0129920.

9. Kankaanpää M, Raitakari M, Muukkonen L, Gustafsson S, Heitto M, Palomäk A, Suojanen K, Harjola V. Use of point-of-care testing and early assessment model reduces length of stay for ambulatory patients in an emergency department. Scand J Trauma Resusc Emerg Med. 2016;24:125.

10. Jarvis P, Davies T, Mitchel K, Taylor I, Baker M. Does rapid assessment shorten the amount of time patients spend in the emergency department? Br J Hosp Med. 2014;75(11):648-51.

11. Larsson A, Greig-Pylypczuk R, Huisman A. The state of point-of-care testing: a european perspective. Ups J Med Sci. 2015;120:1-10.

12. Jang J, Shin S, Lee E, Park C, Song K, Singer A. Use of a comprehensive metabolic panel point-of-care test to reduce length of stay in the emergency department: a randomized controlled trial. Ann Emerg Med. 2013;61(2):145-51.

13. Lee-Lewandrowski E, Corboy D, Lewandrowski K, Sinclair J, McDermot S, Benzer T. Implementation of a point-of-care satellite Laboratory in the Emergency Department of an Academic Medical Center. Arch Pathol Lab Med. 2003;127(4):456-60

14. Singer AJ, Viccellio P, Thode HC Jr, Bock JL, Henry MC. Introduction of a stat laboratory reduces emergency department length of stay. Acad Emerg Med. 2008;15:324-28.
Ready to submit your research? Choose BMC and benefit from:
- fast, convenient online submission
- thorough peer review by experienced researchers in your field
- rapid publication on acceptance
- support for research data, including large and complex data types
- gold Open Access which fosters wider collaboration and increased citations
- maximum visibility for your research: over $100 \mathrm{M}$ website views per year
At BMC, research is always in progress.
Learn more biomedcentral.com/submissions 